\title{
Phase-Noise Reduction of $X$-Band Push-Push Oscillator With Second-Harmonic Self-Injection Techniques
}

\author{
To-Po Wang, Student Member, IEEE, Zuo-Min Tsai, Student Member, IEEE, Kuo-Jung Sun, and \\ Huei Wang, Fellow, IEEE
}

\begin{abstract}
A low phase-noise $X$-band push-push oscillator using proposed feedback topology is presented in this paper. The oscillator core was implemented in a 0.18- $\mu \mathrm{m}$ CMOS process. By using a power splitter and a delay path in the feedback loop connecting the output and current source of the oscillator, a part of the oscillator output power injects to the oscillator itself. With the proper phase delay in the feedback loop and high transconductance of the current source, a low phase-noise oscillator is achieved. The amplitude stability and phase stability are analyzed, the phenomena of the phase-noise reductions are derived, and the device-size selections of the oscillator are investigated. The time-variant function, impulse sensitivity function, is also adopted to analyze the phase-noise reductions of the second-harmonic self-injected push-push oscillator. These theories are verified by the experiments. This self-injected push-push oscillator achieves low phase noise of $-\mathbf{1 2 0 . 1} \mathrm{dBc} / \mathrm{Hz}$ at $1-\mathrm{MHz}$ offset from the 9.6-GHz carrier. The power consumption is $13.8 \mathrm{~mW}$ from a 1.0-V supply voltage. The figure-of-merit of the oscillator is $-188.3 \mathrm{dBc} / \mathrm{Hz}$. It is also the first attempt to analyze the second-harmonic self-injected push-push oscillator.
\end{abstract}

Index Terms-Current source, delay line, figure-of-merit, high- $Q$ resonator, self-injection.

\section{INTRODUCTION}

$\mathbf{T}$ HE increasing demands on wireless data communication have motivated the development of RF front-end circuits toward tens of gigahertz. Being a crucial component in wireless systems, voltage-controlled oscillators (VCOs) impose restrictions on both active and passive devices for the technology of choice. As the CMOS feature size advances to deep submicrometer, CMOS fundamental oscillators at frequencies up to millimeter-wave range were reported [1], [2]. However, it usually requires expensive process technology, while delivering low output power. In order to implement low-cost oscillators for high-frequency applications, a cross-coupled push-push oscillator using a $0.25-\mu \mathrm{m}$ CMOS technology was proposed to achieve an output frequency twice as high as the fundamental frequency with good fundamental rejection, even though the output frequency is higher than the $f_{\max }$ of the transistors [3]-[5]. Though demonstrating high oscillating frequency and

Manuscript received January 30, 2006; revised August 3, 2006. This work was supported in part by the National Science Council under Contract NSC 93-2752-E-002-002-PAE, Contract NSC 93-2219-E-002-024, and Contract NSC 93-2213-E-002-033.

The authors are with the Department of Electrical Engineering and the Graduate Institute of Communication Engineering, National Taiwan University, Taipei, Taiwan 10617, R.O.C. (e-mail: hueiwang@ew.ee.ntu.edu.tw).

Digital Object Identifier 10.1109/TMTT.2006.886912 acceptable output power, the push-push VCO phase noise is usually limited due to lack of high- $Q$ on-chip inductors.

To overcome the poor phase noise in a free-running oscillator, low phase-noise oscillators usually use the high- $Q$ resonators to stabilize the oscillating signals for reducing the noise components [6]-[9]. With a high- $Q$ factor, the VCO will be more stable because the phase fluctuation and frequency fluctuation approximate zero. Injection lock with external stable signal source is another technique used in Si-based circuit design such as frequency dividers [10]-[12] and quadrature generation [13]. With an external injection low-noise signal, the locking range and phase noise of the injection-locked oscillator is associated with the injection signal strength and the $Q$ factor of the resonator of the VCO. The larger the injection signal amplitude, the wider the locking range, and the better the phase noise will be.

The feedback-loop technique is applied in lasers [14], [15] or used to stabilize the oscillators [16]-[18]. The regular self-injection-locked oscillator [19]-[21] is shown in Fig. 1(a) where the self-injection signal has the same frequency as the oscillator. The oscillator output signal goes through the circulator, and then into the input port of the power divider. Part of the oscillator output signal feeds back to the circulator as the self-injection signal. In the feedback loop, the delay cable, high- $Q$ factor resonator, or amplifier, may be used. The phase noise is reduced after self-injection locking as compared to the phase noise of the free-running oscillator while satisfying the stability conditions.

In this paper, a proposed feedback topology is presented. Part of the oscillator output power injects to the current source directly rather than going through the circulator and returning to the oscillator output port. Under the stable oscillation conditions, the feedback push-push signal $\left(2 f_{o}\right)$ can be transformed to a stable signal with larger amplitude to improve the oscillator phase noise. The larger signal injection can be achieved because of the larger transconductance $\left(g_{m 1}\right)$ of the current source rather than the small-signal injection in the reported literatures [19]-[21]. The phase noise of the push-push oscillator using the proposed feedback topology shown in Fig. 1(b), without a circulator and an external amplifier, rivals the phase noise of the push-push oscillator using the regular feedback topology with a circulator shown in Fig. 1(a). The analysis procedures in this paper are based on the methods proposed in [20]-[27]. However, it is the first attempt to analyze the amplitude and phase stability, as well as phase-noise reduction for the proposed second-harmonic self-injected push-push oscillator.

This paper is organized as follows. In Section II, the second-harmonic self-injected oscillator topology is presented, 


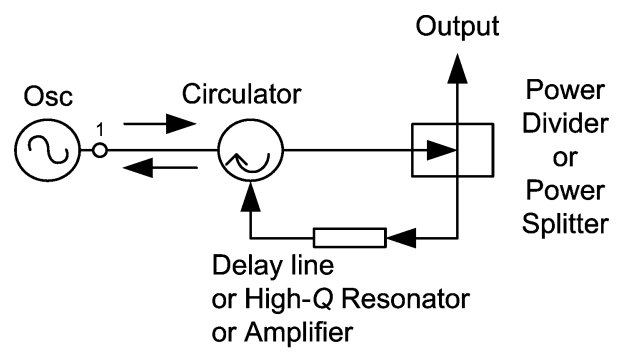

(a)

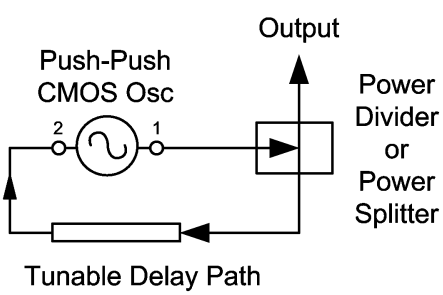

(b)

Fig. 1. Setups for: (a) the regular self-injection-locked oscillator and (b) the proposed self-injected push-push oscillator.

the stability conditions of amplitude fluctuation and phase fluctuation of the fundamental signal $\left(f_{o}\right)$ and push-push signal $\left(2 f_{o}\right)$ are analyzed, and the phenomena of the phase-noise reductions for the push-push signal $\left(2 f_{o}\right)$ are also derived in detail. Section III gives the circuit design method and device-size selections. Section IV gives experimental results and characterization. Section $\mathrm{V}$ gives the conclusions.

\section{Stability Conditions And Phase-Noise AnAlysis of THE PROPOSED CIRCUIT TOPOLOGY}

\section{A. Amplitude Stability Analysis}

The circuit topology of the second-harmonic self-injected push-push oscillator is shown in Fig. 2. The oscillator consists of a cross-coupled pair $\left(M_{2}-M_{3}\right)$, a current source $\left(M_{1}\right)$, a power splitter, and a tunable delay path containing a delay-line cable and a tunable phase shifter. The fundamental frequency $\left(\omega_{o}\right)$ of the oscillator is $1 / \sqrt{L C}$. In order to analyze the behavior of the second-harmonic self-injected oscillator easily, Fig. 3 shows the simplified oscillator model consisting of an $L C$ tank, a conductance $\left(G_{t}\right)$ representing the tank loss, a feedback signal $\left(V_{f}(t)\right)$, and the mildly nonlinear transconductances $\left(g_{m 1}\right.$ to $\left.g_{m 3}\right)$. An oscillator's noise can be modeled by a noise-current source or a complex noise admittance $\left(Y_{n}=G_{n}+j B_{n}\right)$ [24] shown in Fig. 3. $G_{n}$ physically represents the oscillator amplitude fluctuations and $B_{n}$ physically represents the oscillator phase fluctuations. This complex noise admittance $\left(Y_{n}\right)$ is used for the phase-noise reduction analysis.

For the amplitude and phase stability analysis, the dynamic equation for this oscillator shown in Fig. 3 is derived as

$$
\begin{aligned}
C \frac{d^{2}\left(V_{R}-V_{L}\right)}{d t^{2}}+G_{t} \frac{d\left(V_{R}-V_{L}\right)}{d t} & +\frac{1}{L}\left(V_{R}-V_{L}\right) \\
= & g_{m 2} \frac{d V_{R}}{d t}-g_{m 1} \frac{d V_{f}}{d t}
\end{aligned}
$$

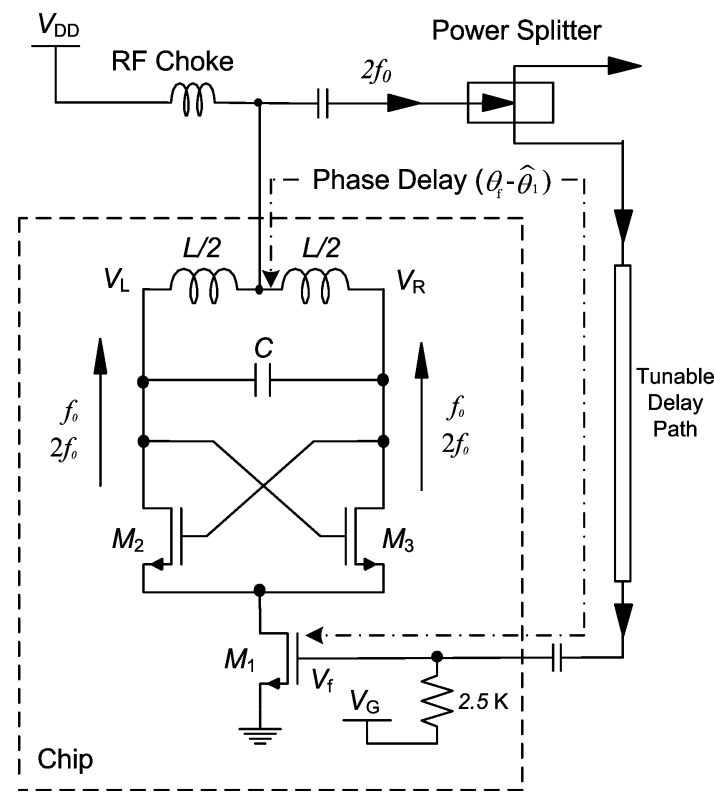

Fig. 2. Circuit topology of the self-injected push-push oscillator.

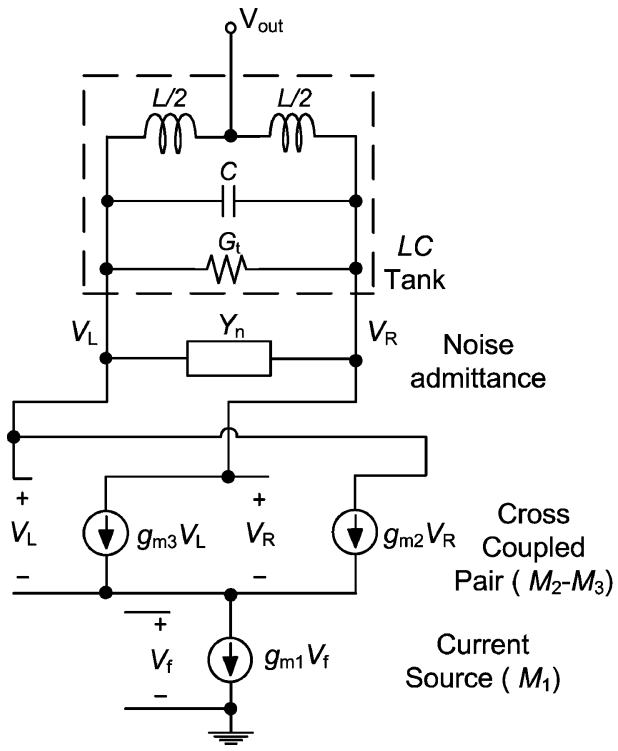

Fig. 3. Simplified push-push oscillator model.

The free-running voltages $\left(V_{L}\right.$ and $\left.V_{R}\right)$ with harmonic terms are assumed time variant and are written as

$$
\begin{aligned}
V_{R}(t)= & \left(A_{o}(t)+\Delta A_{o}(t)\right) e^{j\left(\omega_{o} t+\theta_{o}(t)\right)} \\
& +\left(A_{1}(t)+\Delta A_{1}(t)\right) e^{j\left(2 \omega_{o} t+\theta_{1}(t)\right)}+\cdots \\
V_{L}(t)= & -A_{o}(t) e^{j\left(\omega_{o} t+\theta_{o}(t)\right)}+A_{1}(t) e^{j\left(2 \omega_{o} t+\theta_{1}(t)\right)}+\cdots
\end{aligned}
$$

where $A_{o}(t)$ and $A_{1}(t)$ are the fundamental and second harmonic amplitudes of oscillation, respectively. $\Delta A_{o}(t)$ is the amplitude mismatch of the fundamental signals for $V_{R}(t)$ and $V_{L}(t) . \Delta A_{1}(t)$ is the amplitude mismatch of the second harmonic signals for $V_{R}(t)$ and $V_{L}(t)$. The $\left(\omega_{o}+\theta_{o}(t)\right)$ and $\left(2 \omega_{o}+\theta_{1}(t)\right)$ are the instantaneous phases of fundamental and second harmonic amplitudes of oscillation, respectively. 
For the self-injected push-push oscillator, part of the output signal feeds back to the current source. The current source with the mildly nonlinear transconductance $\left(g_{m 1}\right)$ transforms the feedback signal $\left(V_{f}(t)\right)$ to a larger current format $\left(I_{f}(t)=g_{m 1} \cdot V_{f}(t)\right)$. With the equivalent parallel resistance of the tank $\left(R_{\mathrm{eq}}\right)$ for the second harmonic $\left(2 f_{o}\right)$, the feedback-signal amplitude crossing the tank $\left(V_{\mathrm{inj}}\right)$ is produced $\left(V_{\text {inj }}(t)=I_{f}(t) \cdot R_{\text {eq }}\right)$. This tank amplitude may be larger than the second harmonic amplitude $\left(\left|A_{1}\right|\right)$ generated by the cross-coupled pair $\left(M_{2}-M_{3}\right)$ if the path loss in the feedback loop is small. This situation is $\left|V_{\text {inj }}\right| \geqslant\left|A_{1}\right|$ rather than the low-power injection $\left(\left|V_{\mathrm{inj}}\right|<\left|A_{1}\right|\right)$ in [20] and [21]. The dynamic amplitude behaviors are not considered in [20] and [21] due to the low-power injection. However, the dynamic amplitude behaviors of this study are needed to be considered because of the large-signal injection $\left(V_{\mathrm{inj}}\right)$.

For the push-push oscillator in Fig. 3, the differential fundamental signals are almost canceled in the feedback path. Only the second harmonic signal without amplitude mismatch is considered in the feedback path. Other high-order harmonic signals are also neglected due to the relative small amplitudes of oscillations. Therefore, the feedback signal can be written as

$$
V_{f}(t)=\frac{2 A_{1}(t)}{K} e^{j\left(2 \omega_{o} t+\theta_{f}\right)}
$$

where $K$ is the amplitude-attenuation factor of the delay path and $\theta_{f}$ is the constant phase parameter. Substitute (2)-(4) into (1) and separate the real and imaginary parts of the fundamental signal $\left(f_{o}\right)$ and second harmonic signal $\left(2 f_{o}\right)$, respectively. The real and imaginary parts of the dynamic equations for fundamental signal $\left(f_{o}\right)$ can be written as

$$
\begin{aligned}
& C \frac{d^{2}\left(2 A_{o}\right)}{d t^{2}}+G_{t} \frac{d\left(2 A_{o}\right)}{d t}+\left(\frac{1}{L}-C\left(\omega_{o}+\frac{d \theta_{o}}{d t}\right)^{2}\right)\left(2 A_{o}\right) \\
& \quad=g_{m 2} \frac{d A_{o}}{d t} . \\
& 2 C\left(\omega_{o}+\frac{d \theta_{o}}{d t}\right) \frac{d\left(2 A_{o}\right)}{d t}+2 A_{o} \frac{d^{2} \theta_{o}}{d t^{2}}+G_{t}\left(2 A_{o}\right)\left(\omega_{o}+\frac{d \theta_{o}}{d t}\right) \\
& \quad=g_{m 2} A_{o}\left(\omega_{o}+\frac{d \theta_{o}}{d t}\right) .
\end{aligned}
$$

The real and imaginary parts of the dynamic equations for the second harmonic signal $\left(2 f_{o}\right)$ can be derived as

$$
\begin{aligned}
C & \frac{d^{2}\left(\Delta A_{1}\right)}{d t^{2}}-C\left(\Delta A_{1}\right)\left(2 \omega_{o}+\frac{d \theta_{1}}{d t}\right)^{2}+G_{t} \frac{d\left(\Delta A_{1}\right)}{d t}+\frac{\Delta A_{1}}{L} \\
= & g_{m 2} \frac{d A_{1}}{d t}-\frac{2 g_{m 1}}{K}\left(\frac{d A_{1}}{d t}\right) \cos \left(\theta_{f}-\theta_{1}(t)\right) \\
& \quad+\frac{2 g_{m 1}}{K} A_{1} \cdot\left(2 \omega_{o}\right) \sin \left(\theta_{f}-\theta_{1}(t)\right) \\
2 C & \left(2 \omega_{o}+\frac{d \theta_{1}}{d t}\right) \frac{d\left(\Delta A_{1}\right)}{d t}+G_{t}\left(\Delta A_{1}\right) \frac{d^{2} \theta_{1}}{d t^{2}} \\
& +G_{t}\left(\Delta A_{1}\right)\left(2 \omega_{o}+\frac{d \theta_{1}}{d t}\right) \\
= & g_{m 2} A_{1}\left(2 \omega_{o}+\frac{d \theta_{1}}{d t}\right)-\frac{2 g_{m 1}}{K}\left(\frac{d A_{1}}{d t}\right) \sin \left(\theta_{f}-\theta_{1}(t)\right) \\
& -\frac{2 g_{m 1}}{K} A_{1} \cdot\left(2 \omega_{o}\right) \cos \left(\theta_{f}-\theta_{1}(t)\right) .
\end{aligned}
$$

In order to simplify these equations, the amplitude, phase, and phase derivatives are assumed to vary slowly in (5) and (7). Phase is also assumed to vary slowly in (6) and (8). Therefore, the amplitude and phase dynamic equations (5)-(8) become

$$
\begin{aligned}
\frac{d \theta_{o}}{d t}= & 0 \\
\frac{d A_{o}}{d t}= & -\frac{2 G_{t}-g_{m 2}}{4 C} A_{o} \\
\frac{d \theta_{1}}{d t}= & \frac{-3}{8} \cdot\left(2 \omega_{o}\right)-\frac{g_{m 1} R_{L} \cdot\left(2 \omega_{o}\right)}{2 K Q} \frac{A_{1}}{\Delta A_{1}} \sin \left(\theta_{f}-\theta_{1}(t)\right) \\
\frac{d A_{1}}{d t}= & \frac{g_{m 2} A_{1}\left(2 \omega_{o}\right)-G_{t}\left(\Delta A_{1}\right) \cdot\left(2 \omega_{o}\right)}{\frac{2 g_{m 1}}{K} \sin \left(\theta_{f}-\theta_{1}(t)\right)} \\
& -\frac{\frac{2 g_{m 1}}{K} A_{1}\left(2 \omega_{o}\right) \cos \left(\theta_{f}-\theta_{1}(t)\right)}{\frac{2 g_{m 1}}{K} \sin \left(\theta_{f}-\theta_{1}(t)\right)}
\end{aligned}
$$

The $Q$ in (11) is the quality factor defined as $Q=R_{L} / \omega_{o} L$ of the $L C$ tank. $R_{L}$ is the equivalent parallel resistance of the tank for the fundamental signal $\left(f_{o}\right)$. For the amplitude stability analysis, perturbations are characterized as $A_{o} \rightarrow \widehat{A_{O}}+\delta A_{o}, A_{1} \rightarrow$ $\widehat{A_{1}}+\delta A_{1}$ and $\theta_{1} \rightarrow \widehat{\theta_{1}}+\delta \theta_{1}$, where $\widehat{A_{O}}$ and $\left(\widehat{A_{1}}, \widehat{\theta_{1}}\right)$ are the steady-state solutions for (10) and (12), respectively. $\delta A_{o}, \delta A_{1}$, and $\delta \theta_{1}$ are also the amplitude and phase fluctuations of the oscillator, respectively. If these fluctuations are small, (10) and (12) can be linearized around $\widehat{A_{O}}$ and $\left(\widehat{A_{1}}, \widehat{\theta_{1}}\right)$, respectively, and become

$$
\begin{aligned}
\frac{d \delta A_{o}}{d t}= & -\delta A_{o} \frac{2 G_{t}-g_{m 2}}{4 C} \\
\frac{d \delta A_{1}}{d t}= & -\delta A_{1}\left(2 \omega_{o}\right) \\
& \times \frac{-g_{m 2}+\frac{2 g_{m 1}}{K} \cos \left(\theta_{f}-\widehat{\theta_{1}}\right)+G_{t} \frac{\Delta\left(\delta A_{1}\right)}{\delta A_{1}}}{\frac{2 g_{m 1}}{K} \sin \left(\theta_{f}-\widehat{\theta_{1}}\right)} \\
& -\left(2 \omega_{o}\right) \frac{\frac{2 g_{m 1}}{K} \widehat{A_{1}} \sin \left(\theta_{f}-\widehat{\theta_{1}}\right)\left(\delta \theta_{1}\right)}{\frac{2 g_{m 1}}{K} \sin \left(\theta_{f}-\widehat{\theta_{1}}\right)}
\end{aligned}
$$

For the stable amplitude, the amplitude fluctuations (13)-(14) for fundamental signal $\left(f_{o}\right)$ and second harmonic signal $\left(2 f_{o}\right)$ need to approach zero, respectively. The stable condition for (13) is $2 G_{t}>g_{m 2}\left(R_{t} / 2<1 / g_{m 2}\right)$, which means the tank loss $\left(R_{t} / 2\right)$ is smaller than $1 / g_{m 2}$. In order to let (14) approach zero, $\delta \theta_{1}$ is zero by assuming that the second harmonic frequency is stable. Equation (14) can then be simplified as

$$
\frac{d \delta A_{1}}{d t}=-\delta A_{1}\left(2 \omega_{o}\right) \frac{-g_{m 2}+\frac{2 g_{m 1}}{K} \cos \left(\theta_{f}-\widehat{\theta_{1}}\right)+G_{t} \frac{\Delta\left(\delta A_{1}\right)}{\delta A_{1}}}{\frac{2 g_{m 1}}{K} \sin \left(\theta_{f}-\widehat{\theta_{1}}\right)}
$$

$\Delta\left(\delta A_{1}\right)$ is similar to $\delta A_{1}$ in (15). In order to let (15) approach zero, the denominator and numerator need to be simultaneously greater than zero or smaller than zero. The conditions approximate $2 G_{t}>g_{m 2}$ and

$$
2 n \pi<\theta_{f}-\widehat{\theta_{1}}<(2 n+1) \frac{\pi}{2}
$$


where $\theta_{f}-\widehat{\theta_{1}}$ is the phase delay shown in Fig. 2, and $n$ is an integer.

\section{B. Phase-Stability Analysis}

For the phase-stability analysis, (9) and (11) are used to analyze the phase stability of the fundamental signal $\left(f_{o}\right)$ and second harmonic signal $\left(2 f_{o}\right)$ for the self-injected push-push oscillator, respectively. Perturbations are characterized as $\theta_{O} \rightarrow$ $\widehat{\theta_{O}}+\delta \theta_{o}$ and $\theta_{1} \rightarrow \widehat{\theta_{1}}+\delta \theta_{1}$, where $\left(\widehat{\theta_{O}}, \widehat{\theta_{1}}\right)$ are the steady-state solutions and $\left(\delta \theta_{o}, \delta \theta_{1}\right)$ are the phase fluctuations. Equations (9) and (11) can then be written as

$$
\begin{aligned}
\frac{d\left(\delta \theta_{o}\right)}{d t}= & 0 \\
\frac{d\left(\hat{\theta_{1}}+\delta \theta_{1}\right)}{d t}= & \frac{-3}{8} \cdot\left(2 \omega_{o}\right) \\
& -\frac{g_{m 1} R_{L} \cdot\left(2 \omega_{o}\right)}{2 K Q} \frac{\widehat{A_{1}}}{\Delta A_{1}} \cos \left(\theta_{f}-\widehat{\theta_{1}}\right) \cdot \delta \theta_{1} \\
& -\frac{g_{m 1} R_{L} \cdot\left(2 \omega_{o}\right)}{2 K Q} \frac{\delta A_{1}}{\Delta A_{1}} \sin \left(\theta_{f}-\widehat{\theta_{1}}\right)
\end{aligned}
$$

From (17), the phase fluctuation of the fundamental signal $\left(f_{o}\right)$ equals zero. Therefore, fundamental frequency will be phase stable at $f_{o}$. In (18), assuming the phase fluctuations are small, and $\delta A_{1}$ is zero due to amplitude stable, then (18) can be linearized around $\widehat{\theta_{1}}$, and it becomes

$$
\frac{d \delta \theta_{1}}{d t}=-\frac{g_{m 1} R_{L} \cdot\left(2 \omega_{o}\right)}{2 K Q} \frac{\widehat{A_{1}}}{\Delta A_{1}} \cos \left(\theta_{f}-\widehat{\theta_{1}}\right) \cdot \delta \theta_{1} .
$$

In order to stabilize the phase, the phase fluctuation (19) needs to approach zero. The condition is

$$
\begin{aligned}
\cos \left(\theta_{f}-\widehat{\theta_{1}}\right)>0, \quad(-(2 n+1) \pi / 2 & <\theta_{f}-\widehat{\theta_{1}} \\
& <(2 n+1) \pi / 2) .
\end{aligned}
$$

where $\theta_{f}-\widehat{\theta_{1}}$ is the feedback phase delay shown in Fig. 2, and $n$ is an integer. From (19), with a larger transconductance $\left(g_{m 1}\right)$ of current source, a larger equivalent parallel resistance of the tank $\left(R_{L}\right)$ for the fundamental signal $\left(f_{o}\right)$, a lower amplitude-attenuation factor $(K)$ of the delay path, smaller amplitude imbalance $\left(\Delta A_{1}\right)$ for the second harmonic signal, and higher second harmonic amplitude $\left(\widehat{A_{1}}\right)$, the phase fluctuation of the second harmonic signal $\left(2 f_{o}\right)$ decreases with time more quickly. Therefore, the oscillator will be phase stable.

\section{Phase-Noise Reduction of the Self-Injected Push-Push Oscillator}

For the phase-noise reduction analysis of the push-push signal $\left(2 f_{o}\right)$, the dynamic equations for the amplitude and phase of the self-injected push-push oscillator including the complex noise admittance $\left(Y_{n}=G_{n}+j B_{n}\right)$, shown in Fig. 3, are (21) and (22), shown at the bottom of this page.

$G_{n 1}(t)$ and $B_{n 1}(t)$ in (21) and (22) physically represent the amplitude fluctuation and phase fluctuation of the second harmonic signal $\left(2 f_{o}\right)$, respectively. Assume (21) and (22) are perturbed by $A_{1} \rightarrow \widehat{A_{1}}+\delta A_{1}$ and $\theta_{1} \rightarrow \widehat{\theta_{1}}+\delta \theta_{1}$, where $\left(\widehat{A_{1}}, \widehat{\theta_{1}}\right)$ are the steady-state solutions for (21) and (22), and $\left(\delta A_{1}, \delta \theta_{1}\right)$ are the amplitude and phase fluctuations of the second harmonic signal $\left(2 f_{o}\right)$ for the oscillator, respectively. If these fluctuations are small, $(21)$ and $(22)$ can be linearized around $\left(\widehat{A_{1}}, \widehat{\theta_{1}}\right)$ and become (23) and (24), shown at the bottom of this page. The spectral characteristic of the amplitude and phase fluctuations can be obtained by Fourier transforming (23) and (24). Equations (23) and (24) then become (25) and (26), shown at the bottom of the following page.

The tilde $(\sim)$ presents the variables in the transformed or spectral domain, and $\omega$ is the noise frequency measured relative to the carrier. In (25), the first term on the right-hand side represents the AM-to-AM noise, and the second term represents the conversion of PM noise to AM noise. In (26), the first term on the right-hand side represents the PM-to-PM noise, and the

$$
\begin{aligned}
\frac{d A_{1}}{d t}= & \frac{g_{m 2}\left[\left(A_{1}+\Delta A_{1}\right) \omega_{o}\right]-\frac{2 g_{m 1}}{K} A_{1}\left(2 \omega_{o}\right) \cos \left(\theta_{f}-\theta_{1}(t)\right)}{\frac{2 g_{m 1} 1}{K} \sin \left(\theta_{f}-\theta_{1}(t)\right)+2 B_{n 1}(t)} \\
& -\frac{2 c\left(2 \omega_{o}\right) \frac{d\left(\Delta A_{1}\right)}{d t}+\left(G_{t}+G_{n 1}(t)\right)\left(\Delta A_{1}\right)\left(2 \omega_{o}\right)+B_{n 1}(t) \frac{d\left(\Delta A_{1}\right)}{d t}}{\frac{2 g_{m 1}}{K} \sin \left(\theta_{f}-\theta_{1}(t)\right)+2 B_{n 1}(t)} \\
\frac{d \theta_{1}}{d t}= & -\frac{3+\left(2 \omega_{o}\right) L B_{n 1}(t)+\frac{2 g_{m 1} L}{K}\left(2 \omega_{o}\right) \sin \left(\theta_{f}-\theta_{1}(t)\right) \frac{A_{1}}{\Delta A_{1}}}{\frac{4}{\omega_{o}}+L B_{n 1}(t)}
\end{aligned}
$$

$$
\begin{aligned}
\frac{d \delta A_{1}}{d t} & =\frac{\delta A_{1}\left(g_{m 2} \omega_{o}-\frac{2 g_{m 1}}{K}\left(2 \omega_{o}\right) \cos \left(\theta_{f}-\widehat{\theta_{1}}\right)\right)}{\frac{2 g_{m 1}}{K} \sin \left(\theta_{f}-\widehat{\theta_{1}}\right)}+\frac{\delta \theta_{1} \cdot \widehat{A_{1}} \frac{2 g_{m 1}}{K}\left(2 \omega_{o}\right) \sin \left(\theta_{f}-\widehat{\theta_{1}}\right)}{\frac{2 g_{m 1}}{K} \sin \left(\theta_{f}-\widehat{\theta_{1}}\right)}-\frac{\delta A\left(G_{t}+G_{n 1}(t)\right)\left(2 \omega_{o}\right)}{\frac{2 g_{m 1}}{K} \sin \left(\theta_{f}-\widehat{\theta_{1}}\right)} \\
\frac{d \delta \theta_{1}}{d t} & =\frac{-\left(2 \omega_{o}\right) L B_{n 1}(t)+\delta \theta_{1}(t) \cdot \frac{2 g_{m 1} L}{K}\left(2 \omega_{o}\right) \cos \left(\theta_{f}-\theta_{1}(t)\right) \frac{\widehat{A_{1}}}{\Delta \widehat{A_{1}}}}{\frac{4}{\omega_{o}}+L B_{n 1}(t)}-\frac{\frac{2 g_{m 1} L}{K}\left(2 \omega_{o}\right) \sin \left(\theta_{f}-\theta_{1}(t)\right) \frac{\delta A_{1}}{\Delta\left(\delta A_{1}\right)}}{\frac{4}{\omega_{o}}+L B_{n 1}(t)}
\end{aligned}
$$


second term on the right-hand side represents the conversion of AM-to-PM noise. For a practical oscillator, the amplitude fluctuation is significantly attenuated by the amplitude limiting mechanism. Therefore, the AM-to-PM noise conversion is negligible in the phase-noise analysis and focus here will be on PM-to-PM noise. As a result, the phase fluctuations (26) of the push-push oscillator can be arranged as

$$
\widetilde{\delta \theta_{1}}(\omega)=\frac{\widetilde{B_{n 1}}(\omega)}{\frac{\widehat{A_{1}}}{\Delta \widehat{A_{1}}} \frac{2 g_{m 1}}{K} \cos \left(\theta_{f}-\widehat{\theta_{1}}\right)-j \frac{\omega}{\omega_{3 \mathrm{~dB}} R_{L}}}
$$

where $\omega_{3 \mathrm{~dB}}=\omega_{0} / 2 Q$, half the 3-dB bandwidth of the oscillator tank circuits. The power spectrum of the self-injected push-push oscillator's fluctuations is given by $\left\langle\widetilde{\delta \theta}_{1}{\widetilde{\delta \theta_{1}}}^{*}\right\rangle$, where the notation \langle\rangle represents an ensemble average. The power spectral density of the oscillator's phase fluctuation (i.e., phase noise) is then

$$
\left|\widetilde{\delta \theta_{1}}(\omega)\right|^{2}=\frac{\left|\widetilde{B_{n 1}}(\omega)\right|^{2}}{\left(\frac{\widehat{A_{1}}}{\Delta \widehat{A_{1}}} \frac{2 g_{m 1}}{K} \cos \left(\theta_{f}-\widehat{\theta_{1}}\right)\right)^{2}+\left(\frac{\omega}{\omega_{3 \mathrm{~dB}} R_{L}}\right)^{2}} .
$$

When the oscillator is in the free-running state without selfinjection feedback current $\left(I_{f}=g_{m 1} \cdot V_{f}\right)$. The transconductance $\left(g_{m 1}\right)$ can be assumed zero in (28) because the $g_{m 1}$ in (28) deals with the feedback signal shown in Fig. 3. The free-running phase noise can be written as

$$
\left|\widetilde{\delta \theta_{1}}(\omega)\right|_{\text {free }}^{2}=\frac{\left|\widetilde{B_{n 1}}(\omega)\right|^{2}}{\left(\frac{\omega}{\omega_{3 \mathrm{~dB}} R_{L}}\right)^{2}}
$$

Substituting (29) into (28), the phase fluctuation (i.e., phase noise) of the oscillator becomes

$$
\left|\widetilde{\delta \theta_{1}}(\omega)\right|^{2}=\frac{\left|\widetilde{\delta \theta_{1}}(\omega)\right|_{\text {free }}^{2}}{\left(\frac{\widehat{A_{1}}}{\Delta \widehat{A_{1}}} \frac{2 g_{m 1}}{K} \cos \left(\theta_{f}-\widehat{\theta_{1}}\right) \cdot \frac{\omega_{3} \mathrm{~dB} R_{L}}{\omega}\right)^{2}+1} .
$$

The different phase delays $\left(\theta_{f}-\widehat{\theta_{1}}\right)$ in (30) are considered separately as follows.

1) Phase-Delay Loop $\left(\theta_{f}-\widehat{\theta_{1}}\right)$ Approximate to $2 n \pi$ : While the oscillator is in the self-injection state with the phase-delay loop, $\left(\theta_{f}-\widehat{\theta_{1}}\right)$, shown in Fig. 2, approximates to $2 n \pi$, where $n$ is an integer. From (16) and (20), the oscillator is stable. From (30), the power spectral density of the oscillator's phase fluctu- ation (i.e., phase noise) becomes

$$
\left|\widetilde{\delta \theta_{1}}(\omega)\right|^{2}=\frac{\left|\widetilde{\delta \theta_{1}}(\omega)\right|_{\text {free }}^{2}}{\left(\frac{\widehat{A_{1}}}{\Delta \widehat{A_{1}}} \frac{2 g_{m 1}}{K} \cdot \frac{\omega_{3 \mathrm{~dB}} R_{\mathrm{L}}}{\omega}\right)^{2}+1}
$$

which can be reduced further by increasing the transconductance $\left(g_{m 1}\right)$ of the current source, increasing the equivalent parallel-resistance of the tank $\left(R_{L}\right)$ for the fundamental signal $\left(f_{o}\right)$, reducing the amplitude-attenuation factor $(K)$ of the delay path, reducing amplitude imbalance $\left(\Delta \widehat{A_{1}}\right)$ for second harmonic signal, and increasing second harmonic amplitude $\left(\widehat{A_{1}}\right)$. From (31), the closer to the carrier frequency, the lower the phase noise can be obtained. However, the phase-noise improvement will be limited if the free-running oscillator already has good phase noise. This is because a low phase-noise oscillator typically has a small half the $3-\mathrm{dB}$ bandwidth of the oscillator tank circuits $\left(\omega_{3} d B=\omega_{o} / 2 Q\right)$ or a high quality factor $(Q)$ of the $L C$ tank. From (31), at the frequencies far from the carrier, the phase noise reduces to that of free-running oscillator noise properties

$$
\lim _{\omega \rightarrow \infty}\left|\widetilde{\delta \theta_{1}}(\omega)\right|^{2}=\left|\widetilde{\delta \theta_{1}}(\omega)\right|_{\text {free }}^{2}
$$

2) Phase-Delay Loop $\left(\theta_{f}-\widehat{\theta_{1}}\right)$ Approximate to $(2 n+1) \pi$ : When the oscillator is in the self-injection state with the phasedelay loop $\left(\theta_{f}-\widehat{\theta_{1}}\right)$, the value of the phase-delay loop is set at approximately $(2 n+1) \pi$, where $n$ is an integer. From (15), (16), (19), and (20), the amplitude and phase fluctuations increase with time, and the amplitude and phase difference are unstable. The phase noise becomes worse than that in the free-running state.

\section{D. $1 / f$ Noise Upconversion in Second-Harmonic Self-Injected Push-Push Oscillator}

The Hajimiri's time-varying phase-noise theory [26] is also adopted to analyze the second-harmonic self-injected push-push oscillator. The impulse sensitivity function (ISF) is dimensionless with period $2 \pi$, which describes how much phase shift results from applying a unit impulse. Since ISF is a periodic function with period $2 \pi$, it can be written in a Fourier series

$$
\Gamma\left(\omega_{o} \tau\right)=\frac{c_{o}}{2}+\sum_{n=1}^{\infty} c_{n} \cos \left(n \omega_{o} \tau+\theta_{n}\right)
$$

where the $c_{n}$ terms are the real-value coefficients, and $\theta_{n}$ is the phase of the $n$th harmonic. With the Fourier coefficients in (33),

$$
\begin{aligned}
j \omega \widetilde{\delta A_{1}} & =-\frac{\widetilde{\delta A_{1}}\left(\frac{2 g_{m 1}}{K}\left(2 \omega_{o}\right) \cos \left(\theta_{f}-\widehat{\theta_{1}}\right)+\left(2 \omega_{o}\right)\left(G_{t}+\widetilde{G_{n 1}}(\omega)\right)-g_{m 2} \omega_{o}\right)}{\frac{2 g_{m 1}}{K} \sin \left(\theta_{f}-\widehat{\theta_{1}}\right)}+\frac{\widetilde{\delta \theta_{1}} \cdot \widehat{A_{1}} \frac{2 g_{m 1}}{K}\left(2 \omega_{o}\right) \sin \left(\theta_{f}-\widehat{\theta_{1}}\right)}{\frac{2 g_{m 1}}{K} \sin \left(\theta_{f}-\widehat{\theta_{1}}\right)} \\
j \omega \widetilde{\delta \theta_{1}} & =\frac{\left(\widetilde{\delta \theta_{1}}\right) \frac{\widehat{A_{1}}}{\Delta \widehat{A_{1}}} \frac{2 g_{m 1} L}{K}\left(2 \omega_{o}\right) \cos \left(\theta_{f}-\widehat{\theta_{1}}\right)-\left(2 \omega_{o}\right) L \widetilde{B_{n 1}}(\omega)}{\frac{4}{\omega_{o}}+L \widehat{B_{n 1}}(\omega)}-\frac{\frac{2 g_{m 1} L}{K} \frac{\widetilde{\delta A_{1}}}{\Delta\left(\widetilde{\delta A_{1}}\right)}\left(2 \omega_{o}\right) \sin \left(\theta_{f}-\widehat{\theta_{1}}\right)}{\frac{4}{\omega_{o}}+L \widehat{B_{n 1}}(\omega)}
\end{aligned}
$$




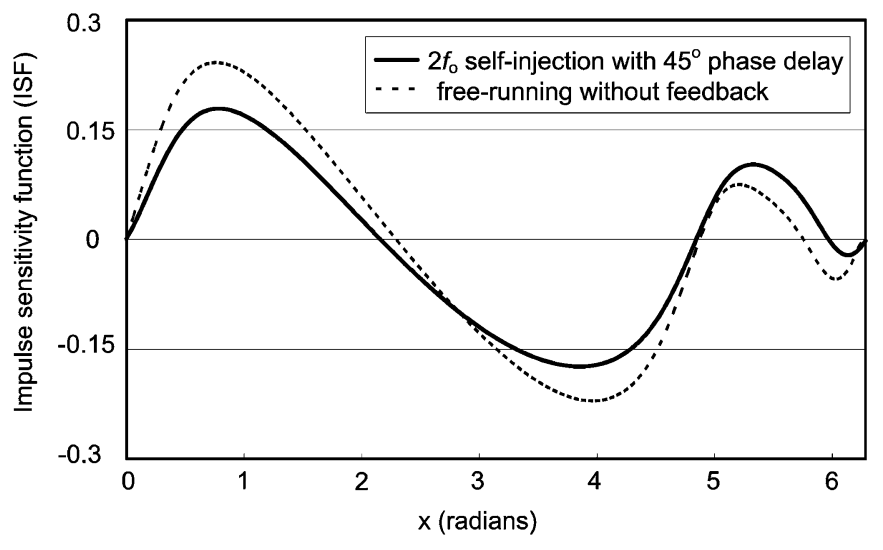

Fig. 4. ISFs of push-push signals $\left(2 f_{o}\right)$ for the free-running push-push oscillator without feedback and the stable self-injected push-push oscillator with $45^{\circ}$ phase delay $\left(\theta_{f}-\widehat{\theta_{1}}\right)$.

the relationship of the device noise $1 / f$ corner frequency and the $1 / f^{3}$ corner in the phase spectrum can be written as [26]

$$
\omega_{1 / f^{3}}=\omega_{1 / f} \cdot \frac{c_{o}^{2}}{2 \Gamma_{\mathrm{rms}}^{2}}
$$

where $c_{o}$ is the twice of the dc value of the ISF and $\Gamma_{\text {rms }}$ is the root mean square value of the ISF. From (34), the $1 / f$ noise upconversion can be significantly reduced by minimizing $c_{o}$. Since $c_{o}$ is twice the dc value of the ISF over a period, reduction of the phase noise in the $1 / f^{3}$ region can be achieved by minimizing the dc value of the ISF [26], [27].

In order to obtain the dc values of the ISFs, the ISFs are calculated using the approximate analytical method in [26]. Although this method is an approximation, it is the easiest to use and rapidly develops important insights into the behavior of an oscillator [26], [27]. Fig. 4 shows the calculated ISFs of the push-push signals $\left(2 f_{o}\right)$ for the free-running push-push oscillator without feedback and the stable second-harmonic self-injected push-push oscillator with $45^{\circ}$ phase delay $\left(\theta_{f}-\widehat{\theta_{1}}\right)$. The absolute dc values for the ISFs of the free-running push-push oscillator without feedback and the stable second-harmonic self-injected push-push oscillator with $45^{\circ}$ phase delay $\left(\theta_{f}-\widehat{\theta_{1}}\right)$ are calculated as 0.0031 and 0.002 , respectively. The absolute ISF dc value of the free-running push-push oscillator is 1.55 times larger than the other one. This means that the free-running push-push oscillator has a larger $1 / f$ noise upconversion factor, leading to a worse phase noise in the $1 / f^{3}$ region. From time-varying phase-noise theory [26], the square of the dc value of the ISF is proportional to the phase noise in the $1 / f^{3}$ region. Therefore, from the ISF calculation, the phase noise in the $1 / f^{3}$ region of the free-running push-push oscillator is approximately $3.8 \mathrm{~dB}$ worse than the self-injected push-push oscillator with $45^{\circ}$ phase delay.

\section{Circuit Design}

\section{A. Oscillator Topology and Phase-Delay Loop Consideration}

The push-push oscillator core was implemented in a commercial $0.18-\mu \mathrm{m}$ bulk CMOS technology. The circuit schematic of the $X$-band self-injected push-push oscillator has been shown in Fig. 2. The oscillator core is composed of an $L C$ tank and a cross-coupled pair with each nMOS of 32 fingers and total gatewidth of $80 \mu \mathrm{m}$. The larger CMOS devices with lower flicker noise were used in the oscillator design. The total gatewidth of the current source $\left(M_{1}\right)$ is $160 \mu \mathrm{m}$.

The negative- $g_{m}$ initiates oscillation at the fundamental frequency $\left(f_{o}\right)$ across the output of the cross-coupled pair along with the harmonic components. The output node is located in the middle of the inductor, behaving as a virtual ground for the differential mode and an open circuit for the common mode with respect to the cross-coupled pair. The out-of-phase fundamental components are cancelled, while the in-phase harmonic components sum up at this node. As a result, an enhanced component at the frequency twice of the $L C$ resonant frequency appears as the oscillator output.

For the self-injected push-push oscillator, a part of the output signal is extracted from the power splitter, then passing through the tunable delay-line path and returning to the gate of the current source. For a stable oscillator, the amplitude fluctuations (15) and phase fluctuations (19) need to approach zero. The amplitude- and phase-stability conditions (16) and (20) have to be satisfied. From (16) and (20), the phase delay shown in Fig. 2 has to be in the range

$$
2 n \pi<\theta_{f}-\widehat{\theta_{1}}<(2 n+1) \frac{\pi}{2}
$$

where $n$ is an integer. From (30), the phase noise can be further reduced near the carrier frequency by using the high-transconductance $\left(g_{m 1}\right)$ current source with $160-\mu \mathrm{m}$ gatewidth, and using the low-loss delay path with low amplitude-attenuation factor $(K)$. A push-push oscillator using a regular self-injection delay path was also designed for comparison.

\section{B. MOS Device Size Consideration}

In the oscillator design, the device size has to be considered. The active devices have influences on the oscillator performances such as phase noise, power consumption, oscillating frequency, and the figure-of-merit. In CMOS design, the MOSFET flicker noise $(1 / f$ noise $)$ at low frequencies generally exhibits a spectral density of the input referred voltage noise $\left(\overline{V_{n}^{2}}\right)$ described in [28, p. 343]

$$
\overline{V_{n}^{2}}=\frac{M}{C_{\mathrm{ox}} W_{g} L_{g}} \cdot \frac{1}{f}
$$

where $M$ is the process-dependent constant, $C_{\mathrm{ox}}$ is the oxide capacitance, $W_{g}$ is the gatewidth, and $L_{g}$ is the gate length. The voltage noise is inversely proportional to the frequency $f$, and the MOS gate area $\left(W_{g} \cdot L_{g}\right)$. Increasing the gate area $\left(W_{g}\right.$. $L_{g}$ ) with constant $W_{g} / L_{g}$ and fixed bias results in lower $1 / f$ noise. However, this will trade off with power consumption and the $1 / f$ noise. In order to reduce the noise contribution to the self-injected push-push oscillator, the $160-\mu \mathrm{m}$ gatewidth nMOS serves as the current source for lower flicker noise $(1 / f$ noise $)$ consideration.

For the cross-coupled pair in the oscillator, the simulated performance with different $0.18-\mu \mathrm{m}$ nMOS gate area $\left(W_{g} \cdot L_{g}\right)$ and different bias conditions are shown in Table I. From this 
TABLE I

Simulated Performance of the Free-RunNing Cross-Coupled OSCILlators With DifFerent Device Sizes AND Bias Conditions

\begin{tabular}{|c|c|c|c|c|c|c|c|}
\hline $\begin{array}{c}\text { Device of Cross- } \\
\text { Coupled Pair } \\
\left(\mathrm{W}_{\mathrm{g}}(\mu \mathrm{m}) / \mathrm{L}_{\mathrm{g}}(\mu \mathrm{m})\right)\end{array}$ & $\begin{array}{c}\text { Device of } \\
\text { Current Source } \\
\left(\mathrm{W}_{\mathrm{g}}(\mu \mathrm{m}) / \mathrm{L}_{\mathrm{g}}(\mu \mathrm{m})\right)\end{array}$ & $\mathrm{V}_{\mathrm{DD}}(\mathrm{V})$ & $\begin{array}{c}\text { Power } \\
\text { Consumption } \\
(\mathrm{mW})\end{array}$ & $\begin{array}{c}\text { Oscillating } \\
\text { Frequency }(\mathrm{GHz})\end{array}$ & $\begin{array}{c}\text { Output } \\
\text { Power } \\
(\mathrm{dBm})\end{array}$ & $\begin{array}{c}\text { Phase Noise } \\
\text { at } 1 \mathrm{MHz} \\
(\mathrm{dBc} / \mathrm{Hz})\end{array}$ & $\begin{array}{c}\text { Figure of Merit } \\
(\mathrm{dBc} / \mathrm{Hz})\end{array}$ \\
\hline \hline $40 / 0.18$ & $160 / 0.18$ & 1.5 & 27 & 10.3 & -2.6 & -117.9 & -180.1 \\
\hline $60 / 0.18$ & $160 / 0.18$ & 1.5 & 28.3 & 9.9 & -1.4 & -116.8 & -182.2 \\
\hline $\mathbf{8 0} / \mathbf{0 . 1 8}$ & $\mathbf{1 6 0 / 0 . 1 8}$ & $\mathbf{1 . 5}$ & $\mathbf{2 8 . 8}$ & $\mathbf{9 . 6}$ & $\mathbf{- 1 . 2}$ & $\mathbf{- 1 1 7 . 5}$ & $\mathbf{- 1 8 2 . 6}$ \\
\hline \hline $40 / 0.18$ & $160 / 0.18$ & 1.0 & 9.9 & 10.4 & -10.5 & -113.5 & -183.8 \\
\hline $60 / 0.18$ & $160 / 0.18$ & 1.0 & 12.3 & 10 & -7.6 & -115 & -184 \\
\hline $\mathbf{8 0 / 0 . 1 8}$ & $\mathbf{1 6 0 / 0 . 1 8}$ & $\mathbf{1 . 0}$ & $\mathbf{1 3 . 9}$ & $\mathbf{9 . 6}$ & $\mathbf{- 6 . 2}$ & $\mathbf{- 1 1 6 . 4}$ & $\mathbf{- 1 8 4 . 6}$ \\
\hline
\end{tabular}

table, using a larger gate-area device in the oscillator cross-coupled pair, lower oscillator phase noise, better figure-of-merit, and higher output power can be obtained under the same bias conditions and the same device-size current sources. However, using a larger gate-area device will consume a little more dc power.

Typically, an oscillator operating at low frequency has better phase noise than that of an oscillator operating at high frequency. A low phase-noise oscillator can be achieved by increasing the power consumption. Therefore, there is a tradeoff between oscillator phase noise and power consumption. In order to evaluate the performance of an oscillator, the figure-of-merit considering the phase noise, carrier frequency, and power consumption is widely adopted in the oscillator papers, e.g., the figure-of-merit is used in [29]-[31], [33], and [39]. The performance of an oscillator evaluated by the figure-of-merit including the phase noise $L$ ( $\left.f_{\text {offest }}\right)$, carrier frequency $\left(f_{o}\right)$, offset frequency $\left(f_{\text {offset }}\right)$, and dc power consumption $\left(P_{\mathrm{DC}}\right)$ can be defined by

$$
L\left(f_{\text {offset }}\right)-20 \log \left(\frac{f_{0}}{f_{\text {offset }}}\right)+10 \log \left(\frac{P_{\mathrm{DC}}}{1 \mathrm{~mW}}\right)
$$

The first and third terms of (37) represent the contributions of phase noise and power consumption to figure-of-merit, respectively. In (37), the values of the figure-of-merit and phase noise are denoted with a negative sign, and the power consumption is denoted with a positive sign. Therefore, the figure-of-merit is linearly proportional to the phase noise, and the figure-of-merit is inversely proportional to the $10 \log \left(P_{\mathrm{DC}} / 1 \mathrm{~mW}\right)$. The phase noise has greater and more direct impact on figure-of-merit than the power consumption $\left(P_{\mathrm{DC}}\right)$ does for a fixed operating frequency $\left(f_{o}\right)$. As a result, from (37) and Table I, using larger gate-area devices in the cross-coupled pair turns out to be a better design approach in this study. Fig. 5 shows the simulated phase noise of the free-running state, unstable state of self-injection, and stable state of self-injection for the push-push signal $\left(2 f_{o}\right)$ of the oscillator with a total $160-\mu \mathrm{m}$ gatewidth current source and a cross-coupled pair with each total $80-\mu \mathrm{m}$ gatewidth nMOS device. The phase noise of the free-running push-push oscillator is improved by using the proposed self-injection feedback loop under stability conditions.

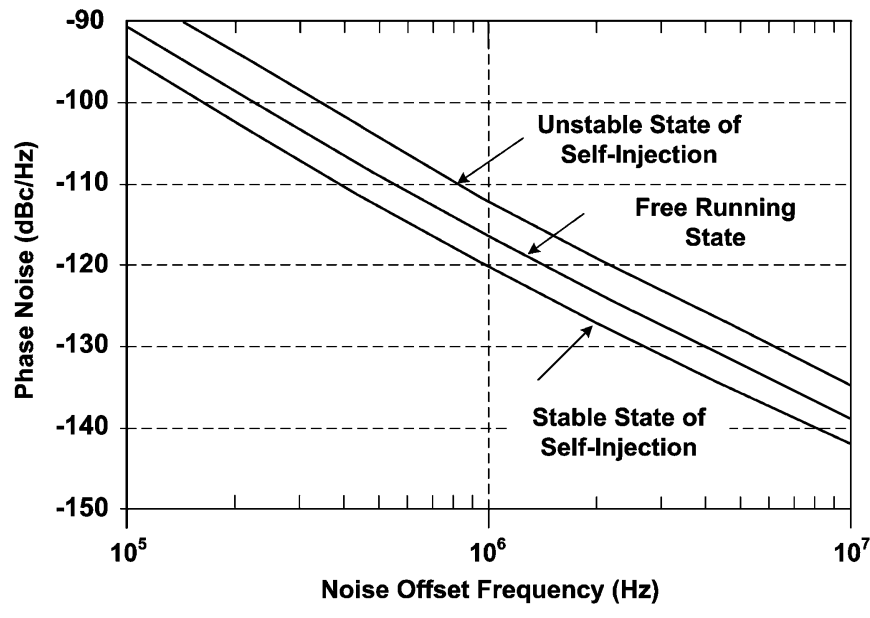

Fig. 5. Simulated phase noise of the free running state, unstable state of selfinjection, and stable state of self-injection for the push-push signal $\left(2 f_{o}\right)$ of this oscillator.

\section{Inductor Consideration}

The oscillator uses on-chip inductors in the $L C$ tank, and an external RF choke shown in Fig. 2 is used to isolate supply voltage $\left(V_{D D}\right)$ from the feedback path for the push-push signal $\left(2 f_{o}\right)$ for the flexibility consideration. The push-push signal $\left(2 f_{o}\right)$ generated from the cross-coupled pair will go to the oscillator output port where it is almost not affected by the supply voltage $\left(V_{D D}\right)$ because of the high-impedance RF choke.

For a single-chip self-injected push-push oscillator in the future, from [4] and [5], in order to obtain a high impedance looking toward the bias $\left(V_{D D}\right)$ for the second harmonic signal $\left(2 f_{o}\right)$, the bias circuits of the push-push oscillator consist of an RF bypass capacitor and a $\lambda / 4$ coplanar waveguide (CPW) for the push-push signal $\left(2 f_{o}\right)$. For an $X$-band push-push oscillator design shown in Fig. 2, the bias circuit can be implemented with a 4-pF on-chip bypass capacitor and a 5-nH on-chip miniature 3-D inductor [37]. For the 5-nH on-chip inductor, the measured $Q$ factor is 6.2 at $10 \mathrm{GHz}$, and the self-resonance frequency is $16 \mathrm{GHz}$ [37].

Table II gives the simulated free-running oscillator phase-noise data at $1-\mathrm{MHz}$ offset with respect to different $Q$ factors of a 5-nH on-chip RF-choke inductor. From this table, the phase noise degrades only $0.43 \mathrm{~dB}$ (from -116.17 
TABLE II

SimUlated Free-RunNing OSCILLATOR PHASE NOISE WITH RESPECT TO $Q$-FACTORS OF THE 5-nH ON-CHIP RF-CHOKE INDUCTOR

\begin{tabular}{|c|c|}
\hline$Q$-Factor & $\begin{array}{c}\text { Oscillator Phase Noise } \\
\text { at 1-MHz Offset }(\mathrm{dBc} / \mathrm{Hz})\end{array}$ \\
\hline \hline 4 & -115.74 \\
\hline 6 & -116.06 \\
\hline 8 & -116.11 \\
\hline 10 & -116.14 \\
\hline 12 & -116.16 \\
\hline 14 & -116.17 \\
\hline
\end{tabular}

to $-115.74 \mathrm{dBc} / \mathrm{Hz}$ ) when the $Q$ factor reduces by 10 (from 14 to 4). In addition, the oscillator phase noise with respect to different $Q$ factors of the $0.7-\mathrm{nH}$ on-chip inductor of the $L C$ tank used in this design is also investigated. The simulated free-running oscillator phase noise degrades $6.7 \mathrm{~dB}$ (from -117.9 to $-111.2 \mathrm{dBc} / \mathrm{Hz}$ ) when the $Q$ factors of the $0.7-\mathrm{nH}$ inductor reduce by 10 (from 14 to 4 ).

As a result, the phase noise of the self-injected push-push oscillator is dominated by the $Q$ factor of the $0.7-\mathrm{nH}$ on-chip inductor of the $L C$ tank. The simulated $Q$ factor of the $0.7-\mathrm{nH}$ on-chip inductor is 14.5 at $10 \mathrm{GHz}$ in this design. For an oscillator with on-chip RF choke in the future, the simulated phase noise of the free-running push-push oscillator with fully integrated on-chip RF choke approximates $-116 \mathrm{dBc} / \mathrm{Hz}$ at $1-\mathrm{MHz}$ offset, and this value is similar to that of the free-running push-push oscillator with external RF choke in this design.

\section{Measured Results of the Self-InJected PUSH-PUSH OSCILLATORS}

Fig. 6 shows the photograph of the fabricated $0.18-\mu \mathrm{m}$ push-push CMOS oscillator with a chip size of $0.61 \mathrm{~mm} \times 0.63 \mathrm{~mm}$ including the testing pads. On-wafer probing was performed to characterize the performance of the oscillator. The phase-noise characteristic of the oscillator is measured using the Agilent E4448A spectrum analyzer. The free-running phase noise can be measured directly from the oscillator output without a power splitter and a feedback path or from one of the power splitter's output ports while the other splitter's output port is connected with the $50-\Omega$ load. The measured free-running phase noise is similar for these two cases. The free-running phase noise is $-116.1 \mathrm{dBc} / \mathrm{Hz}$ at $1-\mathrm{MHz}$ offset from the carrier, as shown in Fig. 7. The oscillator supply voltage $\left(V_{D D}\right)$ is $1.0 \mathrm{~V}$, and the dc power consumption is $13.8 \mathrm{~mW}$. The oscillator is self-injected by a part of its output power through the off-chip power splitter, delay-line cable, and tunable phase shifter with the total phase delay $\left(\theta_{f}-\widehat{\theta_{1}}\right)$ shown in Fig. 2 approximating $45^{\circ}$. From (35), the oscillator is in the stable range. The measured output power is $-14 \mathrm{dBm}$ at $9.6-\mathrm{GHz}$ oscillating frequency after calibrating the path loss, and the fundamental rejection is $45 \mathrm{~dB}$.

Fig. 8 shows the measured phase noise from $100 \mathrm{kHz}$ to $10 \mathrm{MHz}$ of the push-push signals $\left(2 f_{o}\right)$ for the free-running push-push oscillator and the stable second-harmonic self-injected push-push oscillator with $45^{\circ}$ phase delay $\left(\theta_{f}-\widehat{\theta_{1}}\right)$.

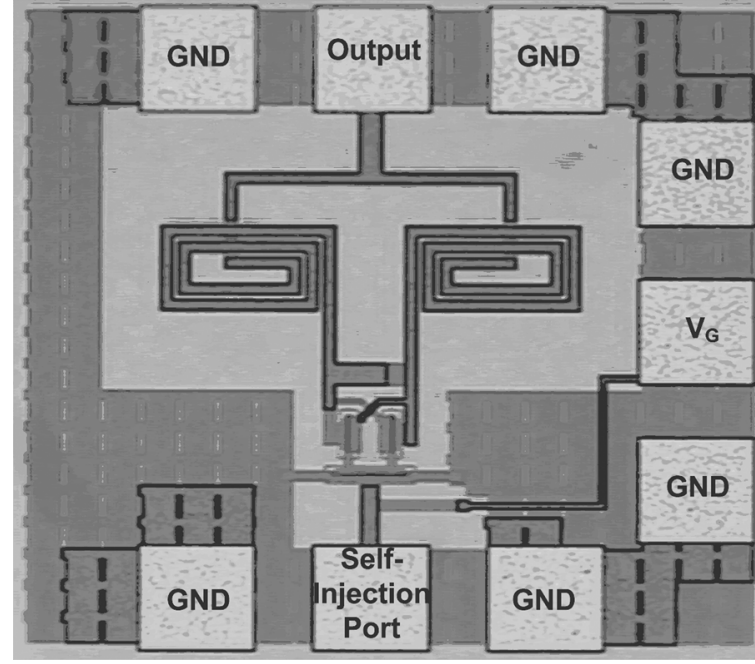

Fig. 6. Chip photograph with the chip size $0.61 \mathrm{~mm} \times 0.63 \mathrm{~mm}$ of the push-push oscillator.

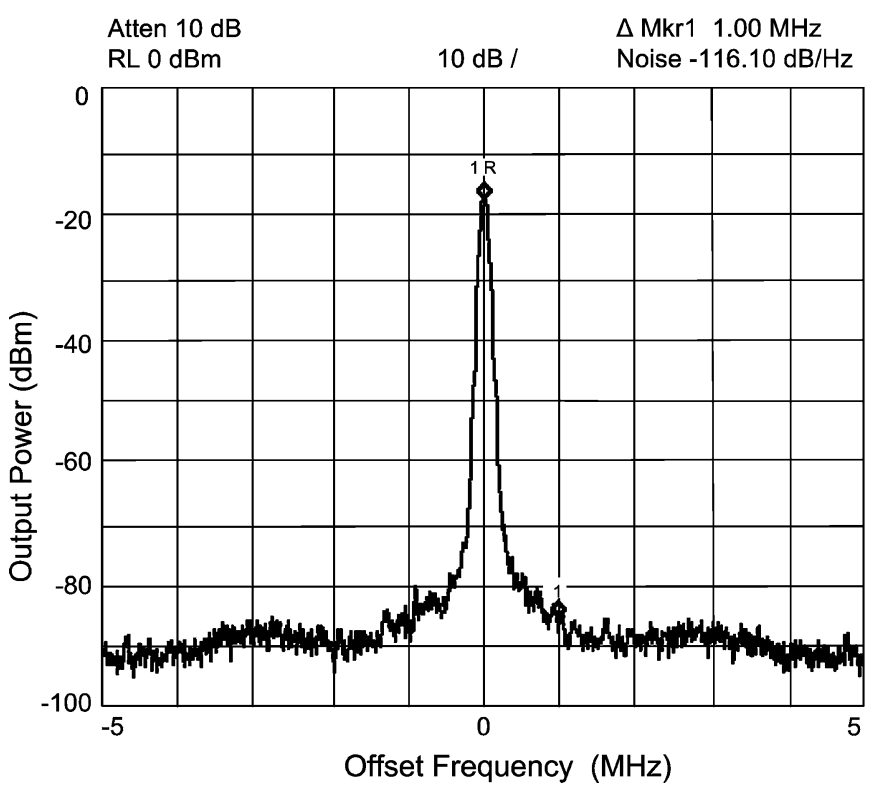

Fig. 7. Measured phase noise of the free-running push-push oscillator at a 9.6-GHz output frequency. $(-116.1 \mathrm{dBc} / \mathrm{Hz}$ at $1-\mathrm{MHz}$ offset from the carrier. The resolution bandwidth is $91 \mathrm{kHz}$.)

From this figure, the $1 / f^{3}$ phase-noise corner frequency is formed at the offset frequency around $1.1 \mathrm{MHz}$ in the self-injected push-push oscillator. The measured phase noise of the self-injected push-push oscillator with $45^{\circ}$ phase delay is improved approximately $4 \mathrm{~dB}$ at $1-\mathrm{MHz}$ offset from the free-running push-push oscillator. The dotted line represents the calculated phase noise in the $1 / f^{3}$ portion for the self-injected push-push oscillator with $45^{\circ}$ phase delay. The calculated phase-noise reduction is approximately $3.8 \mathrm{~dB}$ in the region. The measured phase-noise reduction from $100 \mathrm{kHz}$ to $1 \mathrm{MHz}$ is in agreement with the calculated phase-noise difference. In addition, the measured good $45-\mathrm{dB}$ fundamental rejection for the second-harmonic self-injected push-push oscillator is because of the small amplitude mismatch $\left(\Delta \widehat{A_{O}}\right)$ of the fundamental signals for $V_{R}(t)$ and $V_{L}(t)$ and small 


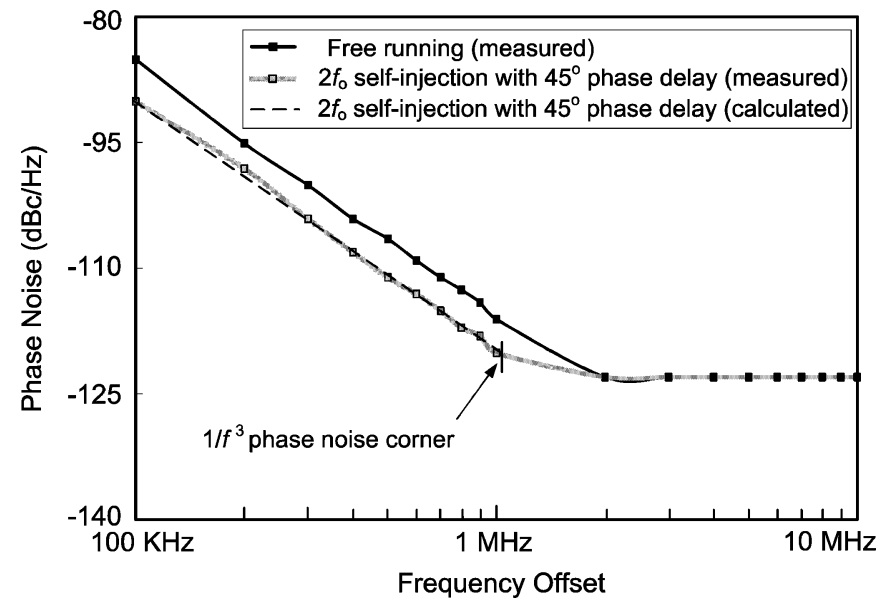

Fig. 8. Phase noise of the free-running push-push oscillator and the secondharmonic self-injected push-push oscillator with $45^{\circ}$ total phase delay.

amplitude mismatch $\left(\Delta \widehat{A_{1}}\right)$ of the second harmonic signals for $V_{R}(t)$ and $V_{L}(t)$. From (30), the small amplitude mismatch $\left(\Delta \widehat{A_{1}}\right)$ of the second harmonic signals and the large second-harmonic oscillation amplitude $\left(\widehat{A_{1}}\right)$ are also the factors resulting in the low phase-noise oscillator. At the same bias condition, the output power is $-16.5 \mathrm{dBm}$ with a phase noise of $-119 \mathrm{dBc} / \mathrm{Hz}$ at $1-\mathrm{MHz}$ offset for the stable self-injected oscillator using a regular delay path and a circulator.

However, if the proposed oscillator is unstable when the total phase-delay $\left(\theta_{f}-\widehat{\theta_{1}}\right)$ shown in Fig. 2 approximates $170^{\circ}$ not in the stable range described in (35), the measured phase noise of the unstable self-injected oscillator is $-111 \mathrm{dBc} / \mathrm{Hz}$ at $1-\mathrm{MHz}$ offset from the carrier. The phase noise degrades approximately $9.1 \mathrm{~dB}$ compared to that of the stable self-injected oscillator $(-120.1 \mathrm{dBc} / \mathrm{Hz}$ at $1-\mathrm{MHz})$ with the same power consumption.

Fig. 9 shows the measured, simulated, and calculated phase noise at $1-\mathrm{MHz}$ offset with respect to the total phase delay $\left(\theta_{f}-\widehat{\theta_{1}}\right)$ illustrated in Fig. 2 of the self-injected push-push oscillator. The simulation tool is Agilent's Advanced Design System (ADS), and the function blocks of the power splitter and phase shifter can be found in the "System-Passive" branch. The calculated phase noise in the $1 / f^{3}$ region is obtained by estimating the phase-noise reduction with usage of the ISF. The measured, simulated, and calculated phase noises are better than $-116.1 \mathrm{dBc} / \mathrm{Hz}$ of the free-running state. In region (I) of Fig. 9, the phase delay is from $0^{\circ}$ to $90^{\circ}$ satisfying the derived amplitude stability condition (16) and derived phase stability condition (20) simultaneously. The measured and simulated phase noises are both better than $-116.1 \mathrm{dBc} / \mathrm{Hz}$ of the free-running state.

In region (II), the total phase delay is from $90^{\circ}$ to $270^{\circ}$. These phase delays do not satisfy both the amplitude stability condition (16) and phase stability condition (20). The measured and simulated phase noise is worse than $-116.1 \mathrm{dBc} / \mathrm{Hz}$ of the free-running state, and a worst $-107.5-\mathrm{dBc} / \mathrm{Hz}$ phase noise occurs at the total phase delay near $180^{\circ}((2 n+1) \pi)$ in this region. These results can also be observed in Section II-C.2.

In region (III), the total phase delay is from $270^{\circ}$ to $360^{\circ}$ satisfying only the phase stability condition (20). The measured

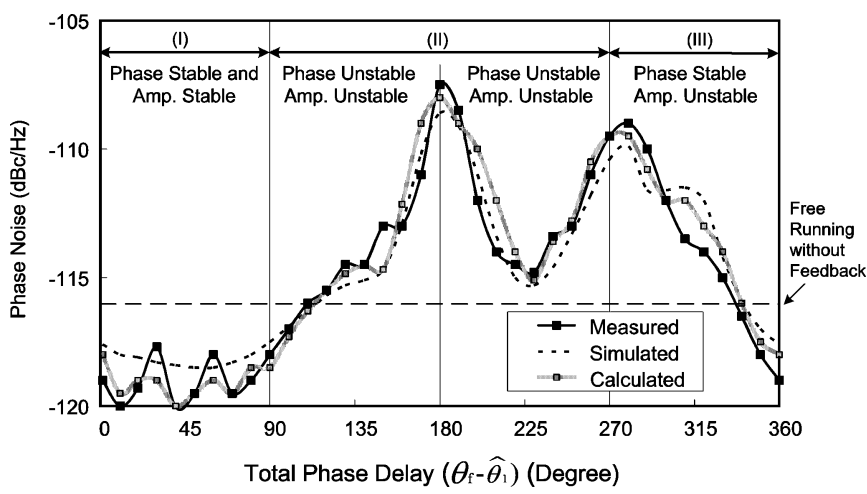

Fig. 9. Measured, simulated, and calculated phase noise with respect to the total phase delays of the self-injected push-push oscillator.

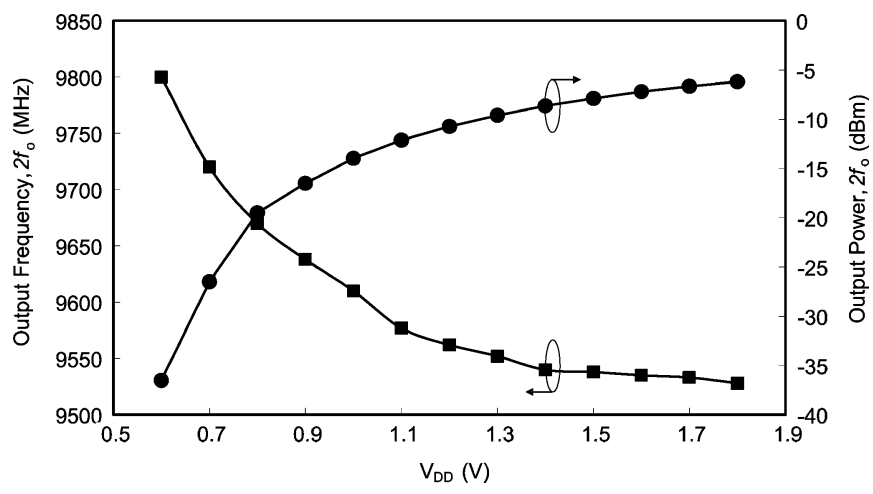

Fig. 10. Measured output frequencies and output powers with respect to the supply voltages $\left(V_{D D}\right)$ of the self-injected push-push oscillator.

and simulated phase noise is almost worse than that of the freerunning state. The total phase delay including the probes, power splitter, delay-line cable, and tunable phase shifter are measured by using the HP8510 network analyzer.

While a 3-dB attenuator is inserted in the feedback loop to increase the amplitude-attenuation factor $(K)$ defined in (30), the measured phase noise degrades $2.9 \mathrm{~dB}$ at $1-\mathrm{MHz}$ offset in the stable self-injection state. When a 6-dB attenuator is inserted in the feedback loop, the phase noise degrades $5.1 \mathrm{~dB}$ at $1-\mathrm{MHz}$ offset in the stable self-injected state. While increasing the transconductance $\left(g_{m 1}\right)$ of the current source by only raising its gate voltage, the oscillator phase noise improved up to $2.8 \mathrm{~dB}$ at $1-\mathrm{MHz}$ offset in the stable self-injection state. These behaviors can be observed from the derived equations (30) and (31).

There is a 3-MHz oscillating frequency difference between the free-running state and the self-injected state under the same bias condition. This is due to the fact that the oscillator output loadings of these two cases are slightly different. Fig. 10 shows the measured output frequencies and output powers with respect to the supply voltages $\left(V_{D D}\right)$ of the self-injected push-push oscillator. The frequency of the second-harmonic self-injected push-push oscillator is tuned by adjusting the supply voltages $\left(V_{D D}\right)$. For the output power higher than $-15 \mathrm{dBm}$, the tuning range is $97 \mathrm{MHz}$ by adjusting the supply voltage $\left(V_{D D}\right)$. While tuning the frequency, the total phase delay can be adjusted to the stable region $\left(2 n \pi<\theta_{f}-\widehat{\theta_{1}}<(2 n+1) \pi / 2\right)$ by tuning the phase shifter to keep low phase noise. The figure-of-merit described in (37) of the free-running push-push oscillator and the 
TABLE III

Performance Summaries of the Recently Reported Si-BASEd Oscillators Around 10 GHz With This Study

\begin{tabular}{|c|c|c|c|c|c|c|c|c|}
\hline Process & $\begin{array}{l}\text { Center } \\
\text { Frequency } \\
(\mathrm{GHz})\end{array}$ & Topology & $\begin{array}{l}\text { Output Power } \\
(\mathrm{dBm})\end{array}$ & $\begin{array}{l}\text { Phase Noise } \\
(\mathrm{dBc} / \mathrm{Hz})\end{array}$ & $\begin{array}{c}\text { Core Power } \\
\text { Consumption } \\
(\mathrm{mW})\end{array}$ & $\begin{array}{l}\text { Supply } \\
\text { Voltage } \\
\text { (V) }\end{array}$ & $\begin{array}{c}\text { Figure of } \\
\text { Merit } \\
(\mathrm{dBc} / \mathrm{Hz})\end{array}$ & Ref. \\
\hline $0.18-\mu \mathrm{m}$ CMOS & 10.2 & Single Balance & -9 & -114.8 at $1 \mathrm{MHz}$ & 15.7 & 1.8 & -183 & [30] \\
\hline $0.18-\mu \mathrm{m}$ CMOS & 11 & $\begin{array}{l}\text { Transformer } \\
\text { Feedback }\end{array}$ & $>-21$ & -109 at $1 \mathrm{MHz}$ & 6.84 & 1.8 & -182 & [31] \\
\hline $0.18-\mu \mathrm{m}$ CMOS & 9.83 & $\begin{array}{l}\text { Cross-Coupled } \\
\text { Complementary }\end{array}$ & Not available & -89 at $0.1 \mathrm{MHz}$ & 5.8 & 1.8 & -181.2 & [32] \\
\hline $0.18-\mu \mathrm{m}$ CMOS & 10 & $\mathrm{LC}$ & -5 & -105 at $1 \mathrm{MHz}$ & 7.2 & 1.8 & -176.4 & [33] \\
\hline $0.13-\mu \mathrm{m}$ CMOS & 10 & $\mathrm{LC}$ & Not available & -95 at $1 \mathrm{MHz}$ & 14.4 & 1.8 & -163.4 & [34] \\
\hline $0.18-\mu \mathrm{m}$ CMOS & 9.39 & $\mathrm{LC}$ & -26 & -120 at $5 \mathrm{MHz}$ & 21.6 & 1.8 & -172.2 & {$[35]$} \\
\hline $\begin{array}{c}0.13-\mu \mathrm{m} \\
\mathrm{CMOS} / \mathrm{SOI}\end{array}$ & 10 & $\mathrm{LC}$ & -18 & -104 at $1 \mathrm{MHz}$ & 26 & 1.2 & -169.8 & [36] \\
\hline 0.18- $\mu \mathrm{m}$ CMOS & 9.6 & $\begin{array}{c}\text { LC } \\
\text { (without } \\
\text { Self Injection ) }\end{array}$ & -14 & -116.1 at $1 \mathrm{MHz}$ & 13.8 & 1.0 & -184.3 & $\begin{array}{l}\text { This Work } \\
\text { free-running }\end{array}$ \\
\hline 0.18- $\mu \mathrm{m}$ CMOS & 9.6 & $\begin{array}{c}\text { Regular } \\
\text { Self Injection }\end{array}$ & -16.5 & -119 at $1 \mathrm{MHz}$ & 13.8 & 1.0 & -187.2 & $\begin{array}{c}\text { This Work } \\
\text { with Circulator }\end{array}$ \\
\hline 0.18- $\mu \mathrm{m}$ CMOS & 9.6 & $\begin{array}{c}\text { Proposed } \\
\text { Second-Harmonic } \\
\text { Self Injection }\end{array}$ & -14 & -120.1 at $1 \mathrm{MHz}$ & 13.8 & 1.0 & -188.3 & $\begin{array}{c}\text { This Work } \\
\text { without Circulator }\end{array}$ \\
\hline
\end{tabular}

stable self-injected push-push oscillator are -184.3 and -188.3 $\mathrm{dBc} / \mathrm{Hz}$, respectively. Table III compares the recently reported Si-based oscillators around $10 \mathrm{GHz}$ with this study. It is observed that the phase noise and figure-of-merit of the free-running push-push oscillator can be improved by using the proposed second-harmonic self-injection technique.

For the proposed second-harmonic self-injected push-push oscillator, with a higher transconductance $\left(g_{m 1}\right)$ of the current source, a higher feedback current $\left(I_{f}=g_{m 1} \cdot V_{f}\right)$ can be obtained. Increasing the feedback current $\left(I_{f}\right)$ can improve the phase noise because of the increased oscillation amplitude $\left(V_{\mathrm{inj}}\right)$ crossing the tank $\left(V_{\mathrm{inj}}=I_{f} \cdot R_{\mathrm{eq}}\right)$, where $R_{\mathrm{eq}}$ is the equivalent parallel resistance of the tank for the $2 f_{o}$ self-injection signal. However, the phase-noise improvement will slow down when the tank amplitude $\left(V_{\mathrm{inj}}\right)$ approaches the oscillator supply voltage $\left(V_{D D}\right)$ shown in Fig. 2.

Since the proposed self-injection topology does not require a circulator, it has the potential for integrating on a single chip in the future. The bias circuit can be implemented with a 4-pF on-chip bypass capacitor and a 5-nH on-chip miniature 3-D inductor [37] for the RF choke. In order to implement a single-chip self-injected push-push oscillator in the future, the delay path variation due to process variation has to be taken into account. An on-chip tunable delay line can be used to alleviate the path variation [38], but an additional process is required. In practical applications, the temperature-independent reference such as bandgap-reference circuits can be used to alleviate the temperature variation.

\section{CONCLUSION}

The design of the low phase-noise second-harmonic self-injected push-push oscillator has been presented. The low phase noise can be achieved when the total phase delay $\left(\theta_{f}-\widehat{\theta_{1}}\right)$ approximates to the stable region $\left(2 n \pi<\theta_{f}-\widehat{\theta_{1}}<(2 n+\right.$ 1) $\pi / 2)$. In addition, with higher transconductance $\left(g_{m 1}\right)$ of the current source, lower amplitude-attenuation factor $(K)$ of the tunable delay path, smaller amplitude mismatch $\left(\Delta A_{1}(t)\right)$ for the second-harmonic signals in $V_{R}(t)$ and $V_{L}(t)$, and larger second-harmonic oscillation amplitude $\left(A_{1}\right)$, a better oscillator phase noise can be obtained. Moreover, using larger device gate area in the oscillator cross-coupled pair, a better oscillator phase noise and figure-of-merit can also be achieved. Furthermore, this second-harmonic self-injected push-push oscillator without a circulator or an amplifier in the feedback path can achieve low phase noise of $-120.1 \mathrm{dBc} / \mathrm{Hz}$ at $1-\mathrm{MHz}$ offset and good figure-of-merit of $-188.3 \mathrm{dBc} / \mathrm{Hz}$.

\section{ACKNOWLEDGMENT}

The authors would also like to thank M.-F. Lei, National Taiwan University, Taipei, Taiwan, R.O.C., for helpful discussion. The authors also thank Prof. George D. Vendelin, Graduate Institute of Communication Engineering, National Taiwan University, Taipei, Taiwan, R.O.C., for editing this paper's manuscript.

The chip was fabricated by TSMC, Hsinchu, Taiwan, R.O.C., through the Chip Implementation Center (CIC), Taiwan, ROC. 


\section{REFERENCES}

[1] H. Wang, "A 50-GHz VCO in 0.25- $\mu \mathrm{m} \mathrm{CMOS,"} \mathrm{in} \mathrm{IEEE} \mathrm{Int.} \mathrm{Solid-}$ State Circuits Conf. Tech. Dig., Feb. 2001, pp. 372-373.

[2] L. M. Franca-Neto, R. E. Bishop, and B. A. Bloechel, "64-GHz and $100-\mathrm{GHz}$ VCOs in 90-nm CMOS using optimum pumping method," in IEEE Int. Solid-State Circuits Conf. Tech. Dig., Feb. 2004, pp. $444-445$.

[3] C. C. Chang, R. C. Liu, and H. Wang, "A 40-GHz push-push VCO using $0.25-\mu \mathrm{m}$ CMOS process," in Asia-Pacific Microw. Conf. Dig., 2003, vol. 1, pp. 73-76.

[4] R. C. Liu, H. Y. Chang, and H. Wang, "A 63-GHz VCO using a standard 0.25- $\mu \mathrm{m}$ CMOS process," in IEEE Int. Solid-State Circuit Conf. Tech. Dig., Feb. 2004, pp. 446-447.

[5] P. C. Huang, M. D. Tsai, H. Wang, C. H. Chen, and C. S. Chang, "A $114-\mathrm{GHz}$ VCO in $0.13-\mu \mathrm{m}$ CMOS technology," in IEEE Int. SolidState Circuit Conf. Tech. Dig., Feb. 2005, pp. 404-406.

[6] Y. T. Lee, J. Lee, and S. Nam, "High- $Q$ active resonators using amplifiers and their applications to low phase-noise free-running and voltage-controlled oscillators," IEEE Trans. Microw. Theory Tech., vol. 52, no. 11, pp. 2621-2626, Nov. 2004.

[7] S. W. Park, "Theoretical verification on the effect of an additional DR in push-push FET DROs," IEEE Microw. Wireless Compon. Lett., vol. 15, no. 11, pp. 466-468, Nov. 2003.

[8] K. Hosoya, K. Ohata, T. Inoue, M. Funabashi, and M. Kuzuhara, "Temperature- and structure-parameters-dependent characteristics of $V$-band heterojunction FET MMMIC DROs," IEEE Trans. Microw. Theory Tech., vol. 51, no. 2, pp. 347-355, Feb. 2003.

[9] F. X. Sinnesbichler, "Hybrid millimeter-wave push-push oscillators using silicon-germanium HBTs," IEEE Trans. Microw. Theory Tech., vol. 51, no. 2, pp. 422-430, Feb. 2003.

[10] H. R. Rategh, H. Samavati, and T. H. Lee, "A CMOS frequency synthesizer with an injection-locked frequency divider for a 5-GHz wireless LAN receiver," IEEE J. Solid-State Circuits, vol. 35, no. 5, pp. 780-787, May 2000.

[11] S. Verma, H. R. Rategh, and T. H. Lee, "A unified model for injectionlocked frequency dividers," IEEE J. Solid-State Circuits, vol. 38, no. 6, pp. 1015-1027, Jun. 2003.

[12] H. R. Rategh and T. H. Lee, "Superharmonic injection-locked oscillator as low power frequency divider," in VLSI Circuits Symp. Tech. Dig., 1998, pp. 132-135.

[13] A. Mazzanti, P. Uggetti, and F. Svelto, "Analysis and design of injection-locked $L C$ dividers for quadrature generation," IEEE J. Solid-State Circuits., vol. 39, no. 9, pp. 1425-1433, Sep. 2004.

[14] K. Hsu and S. Yamashita, "Single-polarization generation in fiber Fabry-Perot laser by self-injection locking in short feedback cavity," J. Lightw. Technol., vol. 19, no. 4, pp. 520-526, Apr. 2001.

[15] S. Yamashita and G. J. Cowle, "Single-polarization operation of fiber distributed feedback lasers by injection locking," J. Lightw. Technol., vol. 17, no. 3, pp. 509-513, Mar. 1999.

[16] H. Kwon and B. Kang, "Linear frequency modulation of voltage-controlled oscillator using delay-line feedback," IEEE Microw. Wireless Compon. Lett., vol. 15, no. 6, pp. 431-433, Jun. 2005.

[17] T. Banky and T. Berceli, "Investigations on noise-suppression effects of nonlinear feed-back loops in microwave oscillators," in IEEE MTT-S Int. Microw. Symp. Dig., May 2004, pp. 2015-2018.

[18] J. E. Rogers and J. Long, "A 10-Gb/s CDR/DEMUX with $L C$ delay line VCO in 0.18- $\mu \mathrm{m}$ CMOS," IEEE J. Solid-State Circuits., vol. 37, no. 12, pp. 1781-1789, Dec. 2002.

[19] K. Kurokawa, "Injection locking of microwave solid-state oscillators," Proc. IEEE, vol. 61, no. 10, pp. 1386-1410, Oct. 1973.

[20] H. C. Chang, "Stability analysis of self-injection-locked oscillator," IEEE Trans. Microw. Theory Tech., vol. 51, no. 9, pp. 1989-1993, Sep. 2003.

[21] H. C. Chang, "Phase noise in self-injection-locked oscillators-theory and experiment," IEEE Trans. Microw. Theory Tech., vol. 51, no. 9, pp. 1994-1999, Sep. 2003.

[22] B. Razavi, "A study of injection locking and pulling in oscillators," IEEE J. Solid-State Circuits., vol. 39, no. 9, pp. 1415-1424, Sep. 2004.

[23] H. C. Chang, X. Cao, M. J. Vaughan, U. K. Mishra, and R. A. York, "Phase noise in externally injection-locked oscillator arrays," IEEE Trans. Microw. Theory Tech., vol. 45, no. 11, pp. 2035-2042, Nov. 1997.

[24] H. C. Chang, X. Cao, U. K. Mishra, and R. A. York, "Phase noise in coupled oscillators: Theory and experiment," IEEE Trans. Microw. Theory Tech., vol. 45, no. 5, pp. 604-615, May 1997.

[25] H. C. Chang, E. S. Shapiro, and R. A. York, "Influence of the oscillator equivalent circuit on the stable modes of parallel-coupled oscillators," IEEE Trans. Microw. Theory Tech., vol. 45, no. 8, pp. 1232-1239, Aug. 1997.
[26] A. Hajimiri and T. H. Lee, "A general theory of phase noise in electrical oscillators," IEEE J. Solid-State Circuits, vol. 33, no. 2, pp. 179-194, Feb. 1998.

[27] J. Choi and A. Mortazawi, "Design of push-push and triple-push oscillators for reducing $1 / f$ noise upconversion," IEEE Trans. Microw. Theory Tech., vol. 53, no. 11, pp. 3407-3414, Nov. 2005.

[28] Y. Tsividis, Operation and Modeling of the MOS Transistor, 2nd ed. Boston, MA: Wiley, 1981

[29] N. Fong, J. Plouchart, N. Zamdmer, D. Liu, L. Wagner, C. Plett, and N. Tarr, "Design of wideband CMOS VCO for multiband wireless LAN applications," IEEE J. Solid-State Circuits., vol. 38, no. 8, pp. 1333-1342, Aug. 2003.

[30] S. Ko, H. D. Lee, D. W. Kang, and S. Hong, "An $X$-band CMOS quadrature balanced VCO," in IEEE MTT-S Int. Microw. Symp. Dig., May 2004, pp. 2003-2006.

[31] N. J. Oh and S. G. Lee, "11-GHz CMOS differential VCO with backgate transformer feedback," IEEE Microw. Wireless Compon. Lett., vol. 15 , no. 11 , pp. 733-735, Nov. 2005.

[32] L. Jia, J. G. Ma, K. S. Yeo, and M. A. Do, "9.3-10.4-GHz-band crosscoupled complementary oscillator with low phase-noise performance," IEEE Trans. Microw. Theory Tech., vol. 52, no. 4, pp. 1273-1278, Apr. 2004.

[33] Z. Gu, B. Bartsch, A. Thiede, R. Tao, and Z. G. Wang, "Fully integrated $10 \mathrm{GHz}$ CMOS LC VCOs," in 33rd Eur. Microw. Conf., 2003, pp. 583-586.

[34] S. Li, J. Kipnis, and M. Ismail, "A 10-GHz CMOS quadrature $L C$-VCO for multirate optical applications," IEEE J. Solid-State Circuits., vol. 38, no. 10, pp. 1626-1634, Oct. 2003.

[35] W. Z. Chen, C. L. Kuo, and C. C. Liu, "10 GHz quadrature-phase voltage controlled oscillator and prescaler," in Eur. Solid-State Conf., 2003, pp. 361-364.

[36] D. Axelrad, E. D. Foucauld, M. Boasis, P. Martin, P. Vincent, M. Belleville, and F. Gaffiot, "A multi-phase $10 \mathrm{GHz} \mathrm{VCO}$ in CMOS/SOI for $40 \mathrm{Gbits} / \mathrm{s}$ SONNET OC-768 clock and data recovery circuits," in IEEE RFIC Symp., 2005, pp. 573-576.

[37] C. C. Tang, C. H. Wu, and S. I. Liu, "Miniature 3-D inductors in standard CMOS process," IEEE J. Solid-State Circuits, vol. 37, no. 4, pp. 471-480, Apr. 2002

[38] D. Kuylenstierna, A. Vorobiev, P. Kinner, and S. Gevorgian, "Ultrawide-band tunable true-time delay lines using ferroelectric varactors," IEEE Trans. Microw. Theory Tech., vol. 53, no. 6, pp. 2164-2170, Jun. 2005.

[39] H. Kim, S. Ryu, Y. Chung, J. Choi, and B. Kim, "A low phase-noise CMOS VCO with harmonic tuned LC tank," IEEE Trans. Microw. Theory Tech., vol. 54, no. 7, pp. 2917-2924, Jul. 2006.

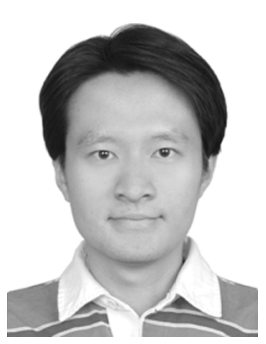

To-Po Wang (S'05) was born in Hsinchu, Taiwan, R.O.C., in 1975. He received the B.S. degrees in mechanical engineering and in electrical engineering from National Sun Yat-Sen University, Taiwan, R.O.C., in 1998, the M.S. degree in communication engineering from National Chiao Tung University, Hsinchu, Taiwan, R.O.C., in 2000, and is currently working toward the Ph.D. degree at the Graduate Institute of Communication Engineering, National Taiwan University, Taipei, Taiwan, R.O.C.

From 2000 to 2003, he was with the BENQ Corporation, Taipei, Taiwan, R.O.C., where he was engaged in mobile phone research. His research interests are in the areas of RF and millimeter-wave integrated circuits (ICs) in CMOS, SiGe BiCMOS, and compound semiconductor technologies.

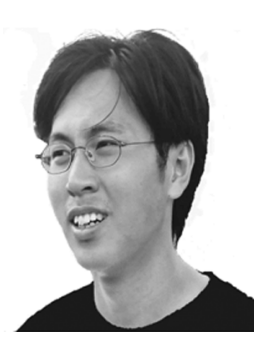

Zuo-Min Tsai (S'01) was born in MaioLi, Taiwan, R.O.C., in 1979. He received the B.S. degree in electrical engineering and $\mathrm{Ph}$.D. degree in communication engineering from National Taiwan University, Taipei, Taiwan, R.O.C., in 2001 and 2006, respectively.

His research interests are the theory of microwave circuits. 


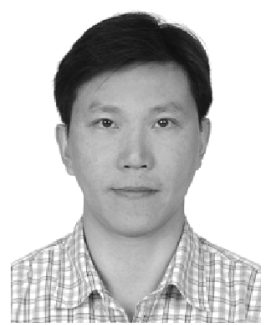

Kuo-Jung Sun was born in Tainan, Taiwan, R.O.C. He received the B.S. degree in electrical engineering from National Tsing Hua University, Hsinchu, Taiwan, R.O.C., in 1991, and the Masters degree in business and administration (MBA) and M.S. degree in communication engineering from National Taiwan University, Taipei, Taiwan, R.O.C., in 1997 and 2005 , respectively.

His research interests include the design of RF integrated circuits (RFICs) and mixed-signal circuits.

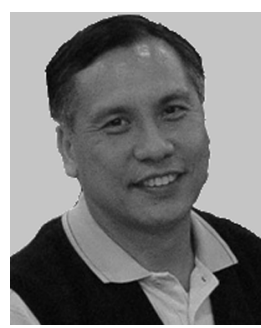

Huei Wang (S'83-M'87-SM'95-F'06) was born in Tainan, Taiwan, R.O.C., on March 9, 1958. He received the B.S. degree in electrical engineering from National Taiwan University, Taipei, Taiwan, R.O.C., in 1980, and the M.S. and Ph.D. degrees in electrical engineering from Michigan State University, East Lansing, in 1984 and 1987, respectively.

During his graduate study, he was engaged in research on theoretical and numerical analysis of electromagnetic radiation and scattering problems. $\mathrm{He}$ was also involved in the development of mi- crowave remote detecting/sensing systems. In 1987, he joined the Electronic Systems and Technology Division, TRW Inc. He has been an MTS and Staff Engineer responsible for monolithic-microwave integrated-circuit (MMIC) modeling of computer-aided design (CAD) tools, MMIC testing evaluation, and design, and then became the Senior Section Manager of the Millimeter-Wave Sensor Product Section, RF Product Center, TRW Inc. In 1993, he visited the Institute of Electronics, National Chiao-Tung University, Hsinchu, Taiwan, R.O.C., to teach MMIC-related topics. In 1994, he returned to TRW Inc. In February 1998, he joined the faculty of the Department of Electrical Engineering, National Taiwan University, as a Professor. He was elected the first Richard M. Hong Endowed Chair Professor of National Taiwan University in 2005.

Dr. Wang is a member of Phi Kappa Phi and Tau Beta Pi. He is a Distinguished Microwave Lecturer for the 2007-2009 term. He was the recipient of the Distinguished Research Award of the National Science Council, R.O.C. (2003-2006). 